\title{
The Unpredictability of Quantum Gravity
}

\author{
S. W. Hawking \\ University of Cambridge, D.A.M.T.P., Cambridge CB3 9EW, England
}

\begin{abstract}
Quantum gravity seems to introduce a new level of unpredictability into physics over and above that normally associated with the uncertainty principle. This is because the metric of spacetime can fluctuate from being globally hyperbolic. In other words, the evolution is not completely determined by Cauchy data at past or future infinity. I present a number of axioms that the asymptotic Green functions should obey in any reasonable theory of quantum gravity. These axioms are the same as for ordinary quantum field theory in flat spacetime, except that one axiom, that of asymptotic completeness, is omitted. This allows pure quantum states to decay into mixed states. Calculations with simple models of topologically non-trivial spacetime indicate that such loss of quantum coherence will occur but that the effect will be very small except for fundamental scalar particles, if any such exist.
\end{abstract}

\section{Introduction}

In the eighteenth and nineteenth centuries it was believed that physics was completely deterministic. That is to say, the classical physical laws determined the exact values of the coordinates and the conjugate momenta of a physical system from their values at one time. In practice, the calculation usually became too complicated for systems of more than a few degrees of freedom, so people resorted to statistical treatments in which they did not attempt to predict the exact state of the system, but only certain gross properties such as the pressure or the temperature. The use of classical statistical mechanics was regarded only as a matter of convenience, however, in principle it was believed that one could predict the exact state of the system.

With the advent of quantum mechanics in the 1920's, it was realized that one could predict exactly either the values of the co-ordinates or the values of the momenta, but not both. More precisely, the most that one could predict exactly were the values of a complete commuting set of observables and that only if the system happened to be in an eigenstate of that set of observables. Thus, roughly speaking, one's ability to make precise predictions was cut in half. As in the case of 
the classical mechanics, it was often useful to employ statistical techniques in which the system was represented by a "mixed state" which was composed of various "pure quantum states" with different probabilities. For a system in such a mixed state, one cannot predict precisely the value of any observable: all one can do is give the probabilities for different values. The use of quantum statistical mechanics was again regarded simply as a matter convenience. In principle one could work always with pure quantum states, because the laws of quantum mechanics or of ordinary quantum field theory were such that a pure state would remain a pure state and would not decay into a mixed state.

The situation changed, however, when it was realized that black holes evaporate by emitting particles with a thermal spectrum [1]. Suppose that one started from an initial pure quantum state which could be described in terms of a complete set of commuting observables on a space-like surface in the past. The same quantum state could also be described in terms of observables in the future only in this case one had to have two sets of observables, observables at infinity which described the outgoing particles and observables inside the black hole which described what fell through the event horizon. The system would still be in a pure quantum state but an observer at infinity could measure only part of the state; he could not even in principle measure what fell into the hole. Such an observer would have to describe his observation by a mixed state which was obtained by summing with equal probability over all the possible black hole states. One could still claim that the system was in a pure quantum state though this would be rather metaphysical because it could be measured only by an angel and not by a human observer.

As the black hole radiated particles, it would lose mass. This would cause the temperature and the rate of emission to increase. When the black hole got down to near the Planck mass, the semi-classical derivation of the thermal emission would break down. We do not have a reliable method of calculating what would happen. There seem to be four main possibilities.

1. The evaporation produces a naked singularity of negative mass which persists.

2. The evaporation slows down and stops leaving a remnant black hole of about the Planck mass.

3. The black hole disappears completely but all the information about the black hole states and any other locally conserved quantities escape to infinity.

4. The black hole disappears completely taking with it the information about the black hole states, and any other quantities which are not coupled to long range fields.

If 1 . were the case, predictability would have broken down completely because one would expect that there would be large numbers of negative mass naked singularities in the universe resulting from the evaporation of black holes of a few Planck masses which would have been formed by thermal fluctuations in the very early stages of the universe [2]. In fact one can probably rule out both possibilities 1. and 2. on observational grounds because otherwise one would expect the mass density of the universe to be dominated by naked singularities or remnant black holes which would give rise respectively to a very large negative or positive deceleration parameter. 
In Case 3 there would be a very large amount of information about the black hole state which would have to be emitted in the final stages. In order to transmit one bit of information in time $\Delta t$ one needs a quantum of frequency $(\Delta t)^{-1}$ with an energy of $h(\Delta t)^{-1}$. However the remaining rest mass energy of the black hole would only be a few Planck masses. Thus the time over which the information was emitted would have to be very long, of order $m_{p}^{-1} \cdot \exp \left\{\frac{4 \pi m^{2}}{m_{p}^{2}}\right\}$, where $m$ is the original mass of the black hole, and $m_{p}$ is the Planck mass. One might therefore have similar difficulties with the deceleration parameter as in Case 2.

Another difficulty with Case 3 would arise if there were some species of massive particle whose number was conserved by a global $U(1)$ symmetry. Even if baryons can decay in some grand unified theory, one could imagine a theory in which baryon number was conserved. Suppose that a star containing about $10^{60}$ baryons collapsed to form a black hole of a few solar masses. During most of the (very long) life of the black hole, its temperature would be very low and most of the rest-mass energy would be emitted in particles of zero rest mass such as neutrinos, photons and gravitons. Only in the final stages would the temperature rise high enough to emit baryons and antibaryons: Even if there was some asymmetry in this emission, there would not be enough energy left to emit more than about $10^{40}$ baryons. Thus it would be impossible for the black hole to disappear completely releasing whatever was inside it.

In view of the above, it seems most reasonable to assume possibility 4 , that the black hole disappears completely taking with it the information about the black hole states and any conserved quantities that are not coupled to long range fields. In this case the initial pure quantum state of the system will have been transformed into a mixed state in which one cannot make any exact predictions about the results of observations, but only give probabilities for the different possible outcomes [3].

If such a decay of a pure quantum state into a mixed state can occur with a macroscopic configuration such as a black hole, it also ought to occur on a microscopic elementary particle level because of quantum fluctuations of the metric which could be interpreted as virtual black holes which appear and disappear again. The aim of this paper is to show how such processes can be described in terms of the Green-functions for quantum gravity. I shall introduce a series of axioms which the Green functions ought to satisfy in any reasonable theory of quantum gravity. These axioms are similar to those that hold for quantum field theory in flat space-time, except that I shall argue that one of the usual axioms, that of asymptotic completeness, does not hold in quantum gravity. Asymptotic completeness means that the asymptotic "in" and "out" states span the Hilbert space of the theory. Asymptotic completeness does not hold in the case of black hole evaporation because the "out" states at infinity do not span the Hilbert space, they have to be supplemented by the black hole states.

If one assumes asymptotic completeness, it can be shown that a pure state will always remain a pure state but, without this axiom, this is not the case. I shall introduce a diagramatic representation for the Green functions which combines the ordinary Feynmann diagrams for the amplitudes with their complex conjugates. The decay of pure states arises from diagrams which cannot be discon- 
nected into a diagram for the amplitude and a complex conjugate diagram. Calculations performed in simple models of topologically non-trivial metrics indicate that such diagrams occur and give estimates of their order of magnitude. They are very small at energies small compared to the Planck energy except for elementary scalar particles. Thus decay of a pure state into a mixed state would not have been observed in laboratory experiments. One would however expect it to have been important in the very early universe.

The decay of a pure state into a mixed state is accompanied by an increase in entropy which does not just arise from course-graining, i.e. from not observing the system as accurately as one could in principle. This would seem to imply that quantum gravity has a built-in arrow of time. However this is not the case. The Green functions of the theory are CPT invariant but the time asymmetry is introduced gy the interpretation: one sets up a system in a pure quantum state initially and then observes what the final state is. Although this process is not CPT invariant in the strong sense that an initial state which was the CPT conjugate of the final mixed state would not evolve to the CPT conjugate of the initial pure state, it may be CPT invariant in a weaker sense of "detailed balance" [3-5]. This would require that the probability of going from a state $A$ to a state $B$ would be the same as the probability of going from the CPT conjugate of $B$ to the CPT conjugate of $A$.

The plan of this paper is as follows: In Sect. 2 I review the formalism for pure and mixed states and I introduce the superscattering operator $\$$ which maps initial density matrices to final density matrices. In Sect. 3 I give a number of axioms for the asymptotic Green functions in quantum gravity which ought to hold in any reasonable theory. Ordinary quantum fields in flat space obey these axioms and an additional axiom, that of asymptotic completeness. If this last axiom holds, the superscattering operator, $\$$, can be factored into a product of an $S$-matrix and its adjoint and pure state would remain a pure state. Section 4 describes a new class of diagrams for the $\$$ operator. These combine a Feynmann diagram for the amplitude with a complex conjugate diagram. If asymptotic completeness does not hold, these diagrams cannot be divided into two disconnected components. In Sect. 5 I review some calculations in simple models of topological fluctuations which indicate that there are diagrams which cannot be disconnected. Section 6 contains a discussion of CPT and global $U(1)$ invariances.

\section{Pure and Mixed States}

I shall adopt the index notation for the components of vectors and tensors on the Hilbert space, $\mathscr{H}$. A ket vector $|\lambda\rangle$ can be expressed as

$$
|\lambda\rangle=\lambda^{A}|A\rangle
$$

where $\{|A\rangle\}$ is a basis for $\mathscr{H}$ and the summation over $A$ is understood. The corresponding bra vector $\langle\lambda|$ in $\overline{\mathscr{H}}$, the complex conjugate Hilbert space, will be denoted by

$$
\langle\lambda|=\lambda_{A}\langle A|
$$


A physical system is described by the density operator, $\varrho$, which can be regarded as a tensor with one index up and one down.

$$
\varrho=\varrho^{A}{ }_{B}|A\rangle\langle B| .
$$

The matrix $\varrho^{A}{ }_{B}$ is hermitian, i.e.

$$
\bar{\varrho}_{A}{ }^{B}=\varrho_{A}^{B} \text {. }
$$

The significance of $\varrho$ can be understood by choosing a basis in which $\varrho^{A}{ }_{B}$ is diagonal, i.e.

$$
\varrho^{A}{ }_{B}=\delta^{A}{ }_{B} P_{A} \quad \text { (no summation on } A \text { ) }
$$

The quantity $P_{A}$ is the probability that the system is in the pure quantum state $|A\rangle$. Thus $Q^{A}{ }_{B}$ must be a positive semi-definite hermitian matrix with trace 1 , i.e.

$$
\varrho_{A}^{A}=1 \text {. }
$$

If one of the probabilities $P_{A}$ is 1 and all the others are zero, the system is said to be in a pure quantum state. Otherwise it is in a mixed state, and the entropy

$$
S=-\sum P_{A} \ln P_{A}
$$

is positive. A pure quantum state may be characterized by $\operatorname{tr}\left(\varrho^{2}\right)=1$, i.e. $\varrho^{A}{ }_{B} \varrho^{B}{ }_{A}=1$ if and only if the state is pure.

I shall consider a situation in which there is an asymptotic region in the past in which the system is described a density operator $\varrho_{-}$on a Hilbert space $\mathscr{H}_{-}$which is a free field Fock space, and an asymptotic region in the future in which the system is described by a density operator $\varrho_{+}$on a future Fock space $\mathscr{H}_{+}$. If one adopts the interpretation that the matrix elements of $\varrho$ in a basis in which it is diagonal correspond to numbers in an ensemble of systems, it follows that $\varrho_{+}$must be a linear function of $\varrho_{-}$. That is to say that there must be a four index tensor $\$$ such that

$$
\varrho_{+}^{A}{ }_{B}=\$_{B C}^{A}{ }^{D} \varrho_{-}{ }^{C} D .
$$

The operator $\$$ is called the superscattering operator and it describes all observations that can be made on the system using only the past and future asymptotic regions. It has to map initial density operators of unit trace to final density operators which are positive, semi-definite and of unit trace. Thus it is hermitian in each of the pairs of indices, $A B$ and $C D$, and

$$
\$_{A C}^{A}=\delta_{C}^{D} \text {. }
$$

This last relation implies the conservation of probability.

In ordinary quantum field theory in flat space-time one also has an operator, $S$, which maps a state $\left|\lambda_{-}\right\rangle$in $\mathscr{H}_{-}$to a state $\left|\lambda_{+}\right\rangle$in $\mathscr{H}_{+}$:

$$
\lambda_{+}{ }^{A}=S^{A}{ }_{B} \lambda^{B} \text {. }
$$

In flat space the $\$$ operator can be expressed as a product of an $S$ operator and its adjoint

$$
\$_{B C}^{A}{ }^{D}=S_{C}^{A} \bar{S}_{B}^{D}
$$


If the factorization (2.11) holds, then $S$ is a unitary operator by (2.9). Also

$$
\begin{aligned}
\operatorname{tr}\left(\varrho^{2}+\right) & =S^{A}{ }_{C} \bar{S}_{B}{ }^{D} \varrho_{-}{ }^{C}{ }_{D} S^{B}{ }_{F} \bar{S}_{A}{ }^{G} \varrho_{-}{ }^{F}{ }_{G} \\
& =\varrho_{-}{ }^{C}{ }_{D} \varrho_{-}{ }^{D}{ }_{C} \\
& =\operatorname{tr}\left(\varrho_{-}{ }^{2}\right) .
\end{aligned}
$$

Thus if (2.11) holds, the system will remain in a pure state if it is in a pure state initially.

A situation in which mixed states arise naturally is when one has a system that can be divided into two parts which do not interact with each other. In this case the Hilbert space $\mathscr{H}$ of the system is the tensor product $\mathscr{H}_{1} \otimes \mathscr{H}_{2}$ of the Hilbert spaces of the parts 1 and 2 . This means that a state $|\lambda\rangle$ in $\mathscr{H}$ can be expressed as a sum of products of vectors in $\mathscr{H}_{1}$ and $\mathscr{H}_{2}$ :

$$
|\lambda\rangle=\lambda^{A B}\left|A_{1}\right\rangle\left|B_{2}\right\rangle \text {, }
$$

where $\left\{\left|A_{1}\right\rangle\right\}$ and $\left\{\left|B_{2}\right\rangle\right\}$ are bases for $\mathscr{H}_{1}$ and $\mathscr{H}_{2}$, respectively. In this case the pair $A B$ of raised indices act as a single raised index in $\mathscr{H}$. If the system is in the pure state $|\lambda\rangle$, its density operator will be

$$
\varrho=\lambda^{A B} \bar{\lambda}_{C D}\left|A_{1}\right\rangle\left|B_{2}\right\rangle\left\langle C_{1}\right|\left\langle D_{2}\right| .
$$

Suppose now that an observer can measure only part 1 of the system and that he has no knowledge of part 2. In that case all possibilities for part 2 would have equal probability as far as he is concerned. Thus he would describe his observations by a reduced density matrix, $\varrho^{\prime}$ which is obtained from $\varrho$ by contracting over the indices $B$ and $D$ which refer to $\mathscr{H}_{2}$ :

$$
\varrho^{\prime}=\lambda^{A B} \bar{\lambda}_{C B}\left|A_{1}\right\rangle\left\langle C_{1}\right| \text {. }
$$

In general $\varrho^{\prime}$ will describe a mixed state on $\mathscr{H}_{1}$ even though $\varrho$ describes a pure state on $\mathscr{H}$.

The situation with black hole evaporation is just of this nature. The total Hilbert space $\mathscr{H}$ can be regarded as the tensor product of a Hilbert space $\mathscr{H}_{1}$ defined in terms of particle states at infinity and a Hilbert space $\mathscr{H}_{2}$ representing states inside the black hole. An observer at infinity cannot measure the black hole states and so has to reduce the density operator to one which corresponds to a mixed state.

\section{Green Functions}

In quantum gravity there is no gauge invariant meaning that can be given to a Green function, $G\left(x_{1}, x_{2}, \ldots, x_{n}\right)$ for a quantum field in the case of general points $x_{1}, x_{2}, \ldots, x_{n}$ because there is no invariant way of specifying the positions of the points: they can always be moved by a diffeomorphism or gauge transformation. However the Green functions can have an invariant meaning in the situation that the metric is asymptotically flat in some suitable sense and the points $x_{1}, x_{2}, \ldots, x_{n}$ all lie in the asymptotic regions. I shall assume that one has some prescription for deriving these asymptotic Green functions and I shall write down a list of some properties they should have in any reasonable theory. Although I shall formulate 
these properties as a series of axioms, I do not intend my treatment to be mathematically rigorous. I believe that it would be possible to give a mathematically precise set of axioms, at least for zero rest mass fields, in terms of null infinity, $\mathscr{I}[6]$. There are some unresolved problems concerning the asymptotic regularity conditions. Although they are important I feel that they are essentially technical in nature and that they do not affect the decay of pure states into mixed states.

I shall assume that there are past and future asymptotic regions which will be referred by suffixes - and + , respectively. I shall assume that one has some theory of quantum gravity which allows one to calculate asymptotic Green functions which are tempered distributions on the past and future asymptotic regions. The Green functions are interpreted as the expectation values of ordered strings of field operators $\left\langle\phi\left(x_{1}\right), \ldots, \phi\left(x_{n}\right)\right\rangle$, where $x_{1}, \ldots, x_{n}$ are in the asymptotic past or future regions and $\phi\left(x_{i}\right)$ denotes a field operator which may have spinor or tensor indices. At this stage I will not specify the Hilbert space on which the operators act nor the state in which the expectation value is taken.

\section{Axiom 1 (Poincaré Invariance)}

Under a Poincaré transformation of the past and future asymptotic regions, the expectation values $\left\langle\phi\left(x_{1}\right) \phi\left(x_{2}\right), \ldots, \phi\left(x_{n}\right)\right\rangle$ transform as they would in Minkowski space.

It may be that one should replace Axiom 1 by invariance under some larger asymptotic group such as the BMS (Bondi-Misner-Sachs) group [7, 8]. However, I feel that this is again a technical point which does not affect the issues under consideration.

\section{Axiom 2 (Hermiticity)}

$$
\left\langle\phi\left(x_{1}\right), \ldots, \phi\left(x_{n}\right)\right\rangle=\left(\left\langle\phi^{+}\left(x_{n}\right), \ldots, \phi^{+}\left(x_{1}\right)\right\rangle\right)^{*},
$$

where $\phi^{+}$is the adjoint operator and * indicates the complex conjugate.

\section{Axiom 3 (Asymptotic Behaviour)}

The Green functions obey the flat space free field wave equations and commutation relations in each asymptotic region, i.e.

$$
\mathscr{L}_{x_{i}}\left\langle\phi\left(x_{1}\right), \ldots, \phi\left(x_{i}\right), \ldots, \phi\left(x_{n}\right)\right\rangle=0,
$$

and if $x_{i}$ and $x_{i+1}$ are in the same asymptotic region, then

$$
\begin{aligned}
& \left\langle\phi\left(x_{1}\right), \ldots, \phi\left(x_{i}\right) \phi\left(x_{i+1}\right), \ldots, \phi\left(x_{n}\right)\right\rangle \mp\left\langle\phi\left(x_{1}\right), \ldots, \phi\left(x_{i+1}\right) \phi\left(x_{i}\right), \ldots, \phi\left(x_{n}\right)\right\rangle \\
& \quad=\left[\phi\left(x_{i}\right), \phi\left(x_{i+1}\right)\right]_{0}\left\langle\phi\left(x_{1}\right), \ldots, \phi\left(x_{i-1}\right) \phi\left(x_{i+2}\right), \ldots, \phi\left(x_{n}\right)\right\rangle,
\end{aligned}
$$

where $\left[\phi\left(x_{i}\right), \phi\left(x_{i+1}\right)\right]_{0}$ is the commutator or anti-commutator of the free field in flat space, and $\mathscr{L}_{x}$ is the flat space free field wave equation.

Axioms 1 and 3 are equivalent to assuming that all interactions between particles are turned off in the infinite past and future. Of course this is not really 
true but it is a convenient fiction just as in ordinary quantum field theory. These axioms also imply that one can represent the field operators in the asymptotic regions in terms of annihilation and creation operators which obey the usual commutation or anti-commutation relations. For instance, a complex scalar field operator $\phi(x)$ and its adjoint $\phi^{+}(x)$ can be represented in the asymptotic past as

$$
\begin{aligned}
\phi(x) & =\frac{1}{(2 \pi)^{3 / 2}} \int \frac{d^{3} \mathbf{k}}{2 k^{0}}\left[a_{-}(k) e^{i k x}+b_{-}^{+}(k) e^{-i k x}\right], \\
\phi^{+}(x) & =\frac{1}{(2 \pi)^{3 / 2}} \int \frac{d^{3} \mathbf{k}}{2 k^{0}}\left[b_{-}(k) e^{i k x}+a_{-}^{+}(k) e^{-i k x}\right] .
\end{aligned}
$$

The operators $a_{-}^{+}(k)$ and $a_{-}(k)$ are the creation and annihilation operators for scalar particles of momentum $k$ in the past. Similarly $b_{-}^{+}(k)$ and $b_{-}(k)$ are operators for antiparticles. They obey the usual commutation relations

$$
\left[a(k), a^{+}\left(k^{\prime}\right)\right]=\left[b(k), b^{+}\left(k^{\prime}\right)\right]=2 k^{0} \delta^{3}\left(\mathbf{k}-\mathbf{k}^{\prime}\right) .
$$

They can be expressed in terms of integrals of the field operators, e.g.

$$
a_{-}(k)=\frac{-i}{(2 \pi)^{3 / 2}} \int_{\Sigma} e^{-i k x} \overleftarrow{\nabla}_{\mu} \phi(x) d \Sigma^{\mu}(x)
$$

where the integral is taken over a space-like or null Cauchy surface for the past asymptotic region. In a similar way one can define creation and annihilation operators in the future asymptotic region. They will obey the usual commutation or anti-commutation relations among themselves but will not have any simple relations with the past operators.

One can use equations like (3.4) to evaluate the expectation values of strings of annihilation and creation operators. The discussion henceforth will be in terms of these strings.

\section{Axiom 4 (Spectral Condition)}

If $Q$ is any string of annihilation and creation operators (they may be in the future or past or a mixture), then $\langle Q\rangle=0$ unless at each point in the string, the sum of the energies of the annihilation operators to the right of that point is less than or equal to the sum of the energies of the creation operators to right of that point, furthermore if the energies are equal, then $\langle Q\rangle=0$ unless the angular momentum and electric charge of the annihilation operators equal those of the creation operators.

In other words, one cannot annihilate more energy than one has already created by acting with creation operators on the right. If this axiom did not hold, one could extract energy from flat space. The provision about angular momentum and electric charge is included because these ought to be conserved by the asymptotic field equations. However quantities that are not coupled to long range fields may not be conserved. This will be discussed in Sect. 6 .

One also has a positivity axiom as in flat space. This can most conveniently be expressed in terms of the annihilation and creation operators for physical particles rather than the Green functions themselves. 


\section{Axiom 5 (Positivity)}

If $Q$ is any sum of strings of annihilation and creation operators, then

$$
\left\langle Q^{+} Q\right\rangle \geqq 0 \text {. }
$$

In other words, the norm of any state is positive.

One can regard Axioms 1-5 simply as statements about the Green functions regarded as a set of distributions but without any interpretation as the expectation values in some state of operators on some Hilbert space. However Axioms 3 and 4 imply that if all the points $x_{i}$ are in the past asymptotic region or if they are all in the future asymptotic region, the Green functions are just the free field Green functions in flat spacetime. One can therefore construct past and future Fock spaces $\mathscr{H}_{-}$and $\mathscr{H}_{+}$with vacuum states $\left|0_{-}\right\rangle$and $\left|0_{+}\right\rangle$so that the purely past or purely future Green functions can be represented as the expectation values of the field operators in the appropriate vacuum states.

One can now define an operator $S$ which maps $\mathscr{H}_{-}$to $\mathscr{H}_{+}$in the following way. Starting from the right, one applies a series of creation operators in the past to create the state $\left|A_{-}\right\rangle$in $\mathscr{H}_{-}$and then one applies a series of annihilation operators in the future which annihilate the state $\left|B_{+}\right\rangle$in $\mathscr{H}_{+}$, i.e.

$$
S_{A}^{B}=\underbrace{\left\langle a_{+}\left(k_{1}\right), \ldots, a_{+}\left(k_{m}\right)\right.}_{B_{+}} \underbrace{a_{-}^{+}\left(l_{1}\right), \ldots, a_{-}^{+}\left(l_{n}\right)}_{A_{-}}\rangle
$$

By Poincaré invariance, $S^{B}{ }_{A}$ will be non-zero only if the states $\left|A_{-}\right\rangle$and $\left\langle B_{+}\right|$have the same energy. One can construct the superscattering operator $\$$ which maps density operators on $\mathscr{H}_{-}$to density operators on $\mathscr{H}_{+}$in a similar manner from the Green functions. Because a density operator is made up of exterior products of ket vectors $\left|A_{-}\right\rangle$and bra vectors $\left\langle B_{-}\right|$, one has to act with a series of creation operators for $\left|A_{-}\right\rangle$and a series of annihilation operators $\left|B_{-}\right\rangle$in the past. The creation operators should appear at the right hand end of the string and the annihilation operators at the left hand end. In between one can put any operator $Q$ whose expectation value one requires in the state with initial density operator $\left|A_{-}\right\rangle\left\langle B_{-}\right|$, i.e.

$$
\operatorname{tr}\left(Q\left|A_{-}\right\rangle\left\langle B_{-}\right)=\langle\underbrace{a_{-}\left(k_{1}\right), \ldots, a_{-}\left(k_{m}\right)}_{B_{-}} Q \underbrace{a_{-}^{+}\left(l_{1}\right), \ldots, a_{-}^{+}\left(l_{n}\right)}_{A_{-}}\rangle .\right.
$$

One can add up the right hand sides of Eq. (3.6) with co-efficients $\varrho_{-}{ }_{B}{ }_{B}$ to obtain the expectation value of the operator $Q$ in the initial mixed state with density operator $\varrho_{-}$

$$
\operatorname{tr}\left(Q \varrho_{-}\right)=\sum \varrho_{-}{ }_{B}{ }_{B}\langle\underbrace{a_{-}\left(k_{1}\right), \ldots, a_{-}\left(k_{m}\right)}_{B_{-}} Q \underbrace{a_{-}^{+}\left(l_{1}\right), \ldots, a_{-}^{+}\left(l_{n}\right)}_{A_{-}}\rangle .
$$

Let the operator $Q$ be of the form $Q_{1} Q_{2}$, where $Q_{1}$ is a string of past creation operators for the state $\left|C_{-}\right\rangle$with energy equal to that of $\left\langle B_{-}\right|$and $Q_{2}$ is a string of past annihilation operators for the state $\left\langle D_{-}\right|$with energy equal to that of $\left|A_{-}\right\rangle$. Then the right hand side of (3.6) is $\delta_{A}{ }^{D} \delta_{B}{ }^{C}$. Thus, by using different operators, $Q$, one can measure the different components of the initial density operator, $\varrho_{-}$. One 
can also find the expectation values of similar operators $Q$ which are made up of annihilation and creation operators in the future, and use them to define the components of a density operator $\varrho_{+}$on $\mathscr{H}_{+}$which corresponds to the initial density operator $\varrho_{-}$on $\mathscr{H}_{-}$. In other words one has defined a linear mapping $\$$ from density operators to density operators whose components are given by

$$
\$_{C A}^{D}{ }^{B}=\langle\underbrace{\mathrm{a}_{-}\left(k_{1}\right), \ldots, a_{-}\left(k_{m}\right)}_{B_{-}} \underbrace{a_{+}^{+}\left(k_{1}^{\prime}\right), \ldots, a_{+}^{+}\left(k_{m^{\prime}}^{\prime}\right.}_{C_{+}}) \underbrace{a_{+}\left(l_{1}^{\prime}\right), \ldots, a_{+}\left(l_{n^{\prime}}^{\prime}\right)}_{D_{+}} \underbrace{a_{-}^{+}\left(l_{1}\right), \ldots, a_{-}^{+}\left(l_{n}\right)}_{A_{-}}\rangle
$$

where the energy of $C$ equals the energy of $B$ and the energy of $D$ equals the energy of $A$.

By Axioms 2 and 5 the dollar operator maps positive semi-definite Hermitian density operators to positive semi-definite Hermitian density operators and by Axiom 4 , it conserves energy, i.e. $\varrho_{+}$cannot contain states with higher energy than $\varrho_{-}$. Conservation of probability however is not guaranteed, so one has to add it as another axiom.

\section{Axiom 6 (Conservation of Probability)}

$$
\$_{A C}^{A}=\delta_{C}^{D} \text {. }
$$

In ordinary quantum field theory, the superscattering operator can be expressed in terms of the $S$ operator by

$$
\$_{B C}^{A}{ }_{B}^{D}=S_{C}^{A} S_{B}^{*}{ }_{B}^{D}
$$

This relation is not guaranteed by Axioms 1-6 but it follows from a further axiom that is normally assumed.

\section{Axiom 7 (Asymptotic Completeness)}

There is a Hilbert space $\mathscr{H}$ with a state $|0\rangle$ and an action of the field operators of on $\mathscr{H}$ such that

(i) the Green functions are the expectation values of the operators in the state $|0\rangle$,

(ii) the asymptotic past operators acting on $|0\rangle$ generate a basis for $\mathscr{H}$. Similarly the asymptotic future operators generate a basis.

Axioms 2 and 5 guarantee that one can find a Hilbert space $\mathscr{H}$ satisfying property (i). Property (ii), however is physically equivalent to the requirement that the expectation values of the field operators should be determined everywhere by their Cauchy data at past or future infinity. One would expect this to be true if and only if the space-time metrics were globally hyperbolic $[3,9]$, which means that the space-times admit one parameter families of Cauchy surfaces. The topology of a globally hyperbolic metric is of the form $\mathbb{R} \times \Sigma$, where $\Sigma$ is the topology of the Cauchy surface. In order to have the right asymptotic behaviour $\sum$ must be $R^{3}$. Thus one would not expect the Green functions of quantum gravity to satisfy Axiom 7 if one allows the possibility of asymptotically flat metrics with topology other than that of $R^{4}$. I shall return to this point in Sect. 5 . 


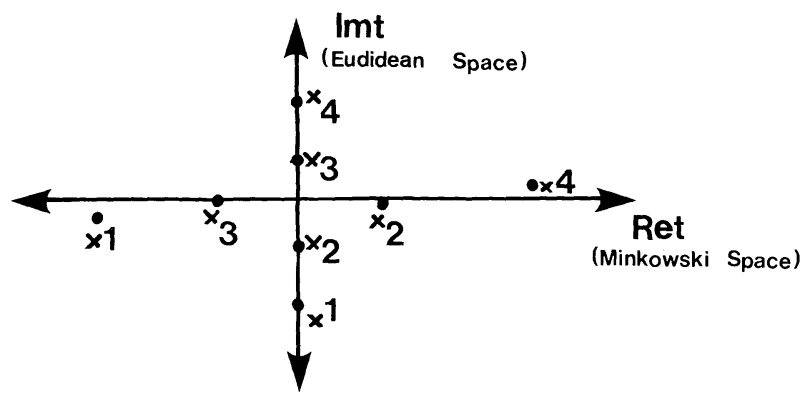

It is straightforward to show that Axiom 7 implies the factorization (3.9) of the $\$$ operator. Insert a summation over a complete set of states, $\sum_{n}|n\rangle\langle n|$ into the
middle of the right hand side of $(3.8)$

$$
\begin{aligned}
{\mathbb{S}^{D}{ }_{C A}^{B}=}^{D} & \sum_{n}\left\langle 0\left|a_{-}\left(k_{1}\right), \ldots, a_{-}\left(k_{m}\right) a_{+}^{+}\left(k_{1}^{\prime}\right), \ldots, a_{+}^{+}\left(k_{m^{\prime}}^{\prime}\right)\right| n\right\rangle \\
& \cdot\left\langle n\left|a_{+}\left(l_{1}^{\prime}\right), \ldots, a_{+}\left(l_{n^{\prime}}^{\prime}\right) a_{-}^{+}\left(l_{1}\right), \ldots, a_{-}^{+}\left(l_{n}\right)\right| 0\right\rangle .
\end{aligned}
$$

By the spectral conditions, Axiom 4, the only states $|n\rangle$ which can contribute to the sum are states of zero energy. But by Axiom 7 the states of $\mathscr{H}$ are in one to one correspondence with the states of the future Fock space $\mathscr{H}_{+}$. The only state in $\mathscr{H}_{+}$with zero energy is $\left|0_{+}\right\rangle$which is in correspondence with the state $|0\rangle$ in $\mathscr{H}$. Thus $\sum_{n}|n\rangle\langle n|$ reduces to $|0\rangle\langle 0|$ and

$$
\$_{C A}^{D}{ }^{B}=S_{A}^{D} S_{C}^{*}{ }^{B}
$$

Thus pure states remain pure states. Conservation of probability (Axiom 6) implies that $S$ is unitary.

\section{Graphical Representation}

The spectral condition (Axiom 4) implies that a Green function $\left\langle\phi\left(x_{1}\right), \ldots, \phi\left(x_{n}\right)\right\rangle$ is a boundary value of a function of the complexified co-ordinates $x_{1}, \ldots, x_{n}$ which is holomorphic in the future tube, i.e. for $x_{i+1}-x_{i}=\alpha+i \beta$, where $\alpha$ and $\beta$ are real 4-vectors in flat space-time, and $\beta$ is a future directed time-like vector. In ordinary quantum field theory in flat space-time one normally assumes local causality, i.e. that the field operators commute (or anticommute) at space-like separated points. With the spectral condition this implies that the Green functions for any operator ordering can be obtained as the boundary values of a single holomorphic function [10]. This holomorphic function can be characterized by its value, $G_{E}\left(x_{1}, \ldots, x_{n}\right)$, in the Euclidean space obtained by choosing the time co-ordinates of each of the points $x_{1}, \ldots, x_{n}$ to be purely imaginary. The required Green functions $\left\langle\phi\left(\mathrm{x}_{1}\right), \ldots, \phi\left(\mathrm{x}_{n}\right)\right\rangle$ in real Minkowski space are then obtained by analytically continuing $G_{E}\left(x_{1}, \ldots, x_{n}\right)$ in the coordinates $x_{1}, \ldots, x_{n}$ while maintaining the imaginary part of each of the differences $x_{i+1}-x_{i}$ to be a future directed timelike vector. This analytic continuation in complex $t$ for the Green function $\left\langle\phi\left(x_{1}\right) \phi\left(x_{2}\right) \phi\left(x_{3}\right) \phi\left(x_{4}\right)\right\rangle$ is shown in Fig. 1. The significance of the requirement 
that $x_{i+1}-x_{i}$ should have an imaginary past which is future directed timelike is that this guarantees that the points $x_{1}, \ldots, x_{n}$ are never null related to each other during the analytic continuation from Euclidean space to Minkowski space. The Green functions in general are holomorphic except for singularities on the complex light cone.

In the situation being considered in this paper where one has only asymptotic Green functions, one cannot really impose local causality as an axiom because a point in the future asymptotic region cannot be spacelike separated from a point in the past region. Instead it seems reasonable to adopt what in flat space is a consequence of local causality.

\section{Axiom 8 (Euclidean Postulate)}

The Green functions for any operator ordering $\left\langle\phi\left(x_{1}\right), \ldots, \phi\left(x_{n}\right)\right\rangle$ are the boundary values of a single holomorphic function $G_{E}\left(x_{1}, \ldots, x_{n}\right)$ defined for points $x_{1}, \ldots, x_{n}$ in the asymptotic regions of Euclidean space.

In order to have a definite theory I shall assume that these Euclidean asymptotic Green functions $G_{E}\left(x_{1}, \ldots, x_{n}\right)$ are obtained by performing a path integral over all asymptotically Euclidean positive definite metrics and all matter fields in them. A metric is said to be asymptotically Euclidean (A.E.) if outside some compact set it approaches the standard Euclidean metric on $R^{4}$ at some suitable rate. The topology of the metric can however be different from that of $R^{4}$.

The path integral over all matter fields in a given positive definite metric can be performed by standard perturbation theory and the Green functions can be represented graphically in the normal way by diagrams in which lines correspond to free field propagators in the given metric and vertices represent interactions. One then averages the resultant Green functions over all positive definite metrics, each weighted by the exponential of minus its gravitational action. We do not know how to perform this averaging over metrics but we might hope to get some idea of qualitative features and orders of magnitudes by averaging over restricted classes of metrics. This will be described in Sect. 5 .

In this manner one obtains a graphical representation for each asymptotic Euclidean Green function $G_{E}\left(x_{1}, \ldots, x_{n}\right)$. One now has analytically to continue $G_{E}\left(x_{1}, \ldots, x_{n}\right)$ to the asymptotic regions of Minkowski space in an appropriate manner to obtain the real space-time Green function with the desired operator ordering. This may be done by choosing a frame in which the initial spacelike coordinates of the points $x_{1}, \ldots, x_{n}$ are real and the timelike coordinates are purely imaginary. One then analytically continues the time coordinates to the neighbourhood of the real $t$-axis but displaces them slightly in imaginary time according to the operator ordering required. For instance, if one wants $\left\langle\phi\left(x_{1}\right), \ldots, \phi\left(x_{n}\right)\right\rangle$, then one chooses points $x_{1}, \ldots, x_{n}$ to have small imaginary time coordinate such that each imaginary time difference $x_{i+1}-x_{i}$ is positive.

One can use the diagrams for the Green functions to give graphical representations of the $S$ and $\$$ operators in the complex $t$ plane. The $S$ operator was given by

$$
S_{A}^{B}=\langle\underbrace{a_{+}\left(k_{1}\right), \ldots, a_{+}\left(k_{m}\right)}_{B_{+}} \underbrace{a_{-}^{+}\left(l_{1}\right), \ldots, a_{-}^{+}\left(l_{n}\right)}_{A_{-}}\rangle .
$$




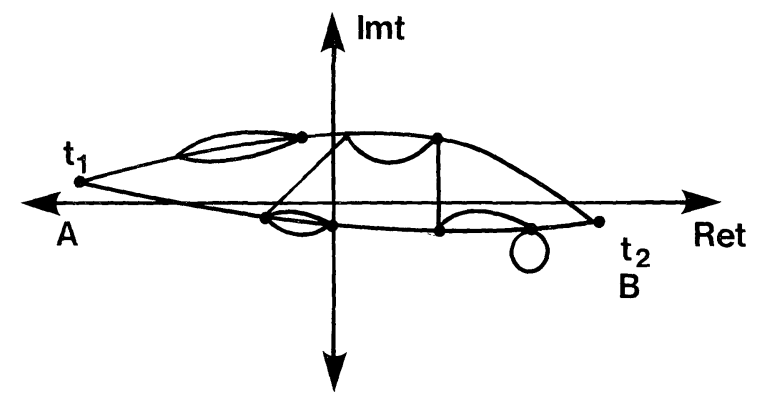

Fig. 2

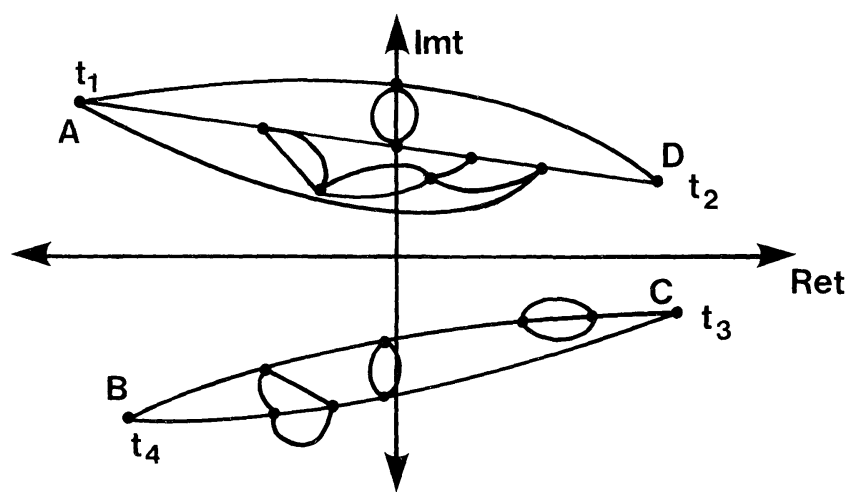

Fig. 3

The creation operators in the past can be considered to be integrals of the field operators over a spacelike hypersurface of constant $t=t_{1}$, where $t_{1}$ has a very large negative real part and a small positive imaginary part (Fig. 2). The creation operators in the past all commute with each other and so can be placed at the same value of imaginary $t$. Similarly the annihilation operators in the future can be defined by integrals of the field operators over a spacelike hypersurface of constant $t=t_{2}$, where $t_{2}$ has a large positive real part and a small negative imaginary part. The lines between $t_{1}$ and $t_{2}$ are topologically equivalent to the graphical representation of $G_{E}\left(x_{1}, \ldots, x_{n}\right)$ in a fixed positive definite metric. No significance is attached to their positions in Fig. 2.

The superscattering operator is

$$
\$_{C A}^{D}{ }^{B}=\langle\underbrace{a_{-}\left(k_{1}\right), \ldots, a_{-}\left(k_{m}\right)}_{B_{-}} \underbrace{a_{+}^{+}\left(k_{1}^{\prime}\right), \ldots, a_{+}^{+}\left(k_{m^{\prime}}^{\prime}\right.}_{C_{+}} \underbrace{a_{+}\left(l_{1}^{\prime}\right), \ldots, a_{+}\left(l_{n^{\prime}}^{\prime}\right)}_{D_{+}} \underbrace{\left.a_{-}^{+}\left(l_{1}\right), \ldots, a_{-}^{+}\left(l_{n}\right)\right\rangle}_{A_{-}} .
$$

In this case one can analytically continue $G_{E}\left(x_{1}, \ldots, x_{n}\right)$ so that the creation operators for the state $A_{-}$in the past are at $t=t_{1}$, where $R_{e}\left(t_{1}\right)=-\infty$, the annihilation operators for the state $D_{+}$in the future are at $t=t_{2}$, where $R_{e}\left(t_{2}\right)=+\infty$ and $\operatorname{Im}\left(t_{2}\right)<\operatorname{Im}\left(t_{1}\right)$, the creation operators for the state $C$ in the future are at $t=t_{3}$, where $\operatorname{Im}\left(t_{3}\right)<\operatorname{Im}\left(t_{2}\right)$ and the annihilation for the state $B$ in the past are at $t=t_{4}$, where $\operatorname{Im}\left(t_{4}\right)<\operatorname{Im}\left(t_{3}\right)$ (Fig. 3). The graph shown in this figure 


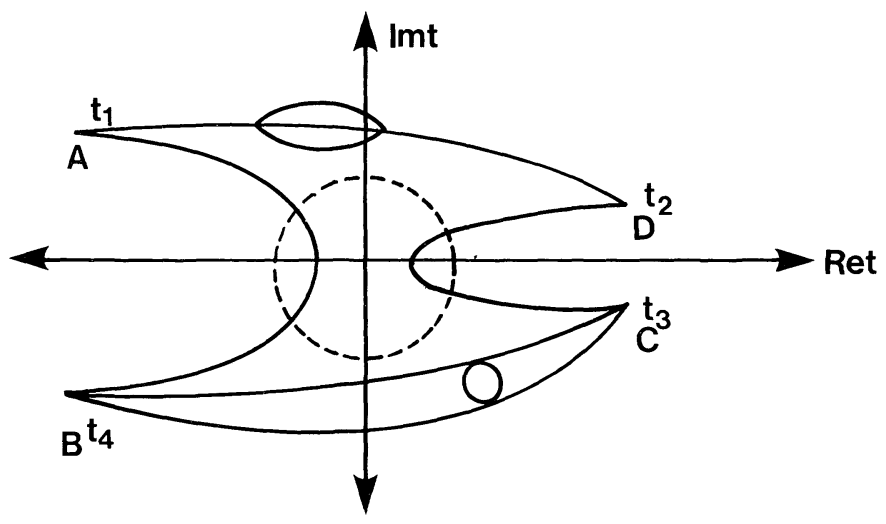

Fig. 4

consists of two parts which are disconnected by the real $t$-axis. The upper part which joins $t_{1}$ to $t_{2}$ is similar to a graph for the $S$ operator while the other part is similiar to a graph for the adjoint $S$ operator. If all the diagrams could be disconnected in this way, the superscattering operator, $\$$, would factor into a product of the $S$ operator and its adjoint as in (3.9). However the calculations described in Sect. 5 indicate that topologically non-trivial metrics give diagrams like those in Fig. 4 which cannot be disconnected. The dotted circle in the middle of the figure represents a topological fluctuation or quantum gravitational bubble which allows lines to go directly from $t_{1}$ to $t_{4}$ and from $t_{2}$ to $t_{3}$. Because of these diagrams, the superscattering operator does not obey the factorization condition (3.9), pure states can evolve into mixed states and the $S$ operator is non-unitary. However, the estimates of section 5 indicate that diagrams such as Fig. 4 are very small at normal energies except for elementary scalar particles. No such particles have been observed yet, so that these predictions are not in conflict with experiment.

\section{Model Calculations}

To calculate the asymptotic Euclidean Green functions, one first calculates them in a given asymptotically Euclidean positive definite metric and then averages the result over metrics with the appropriate weighting. The first step is difficult in general even for matter fields which do not have any interactions except with the gravitational field and we do not know how to carry out the second step. One might hope however that one could get some approximation by averaging over a finite dimensional family of metrics rather than the infinite dimensional space of all metrics. Calculations of this sort have been carried out in simple classes of metrics with the topologies of $S^{2} \times S^{2}$ and $C P^{2}$ with a point removed $[11,12]$. Gross and Witten have also suggested calculations on a metric with topology of $R P^{3} \times R$. The metrics all have zero or self dual Weyl tensor. This makes it easy to find the Green functions for conformally invariant fields because they obey Huygens principle. It is reasonable to consider only zero rest mass fields because 
any rest mass can be regarded as a small perturbation compared to the Planck mass. The averaging over different metrics in the family is straightforward apart from an undetermined measure on the scale factor.

Having obtained the asymptotically Euclidean Green functions, one has to continue them analytically to the Minkowski asymptotic region and integrate them over the appropriate plane waves to obtain the $\$$ operator. The best way of doing this, at least for zero rest mass fields, is to use the concept of null infinity, $\mathscr{I}$, introduced by Penrose [6]. This is a null hypersurface which is attached to the space-time manifold $M$ by a conformal compactification. It may be regarded as the light cone of the point at infinity, I, which can be added to a positive definite asymptotically Euclidean metric by a conformal transformation with a conformal factor, $\Omega$, of order $r^{-2}$. One analytically continues the Euclidean Green functions to the light cone of the point, I, and integrate them with the same Cauchy data that one would use for plane waves in flat space. The $C P^{2}$ metrics used in $[11,12]$ do not approach flatness sufficiently fast at infinity to have a well behaved null infinity. Thus the results from these calculations must be treated with caution. However, the $S^{2} \times S^{2}$ and the $R P^{3} \times R^{1}$ metrics have well behaved null infinities.

The Green functions obtained from the finite dimensional calculations described above satisfy Axiom 1 (Poincaré Invariance) and Axiom 2 (Hermiticity). However they do not satisfy Axiom 3 (Asymptotic Behaviour) which would require that they were just the flat space free-field Green functions if all the points lay on past null infinity, $\mathscr{I}^{-}$, or all on future null infinity, $\mathscr{I}^{+}$. This is because the Green functions in each metric obey Huygens principle and so are meromorphic in the complexified manifold. Their values are therefore the same if all the points are on $\mathscr{I}^{-}$or if some of them are at the corresponding points on $\mathscr{I}^{+}$. In more general metrics the Green functions will have branch cuts which allow different analytic continuations to points on $\mathscr{I}^{-}$and $\mathscr{I}^{+}$. These Green functions also do not satisfy the spectral conditions (Axiom 4). This is because these conditions can be regarded as an expression of the conservation of energy. The conservation of energy at infinity is a consequence of the Einstein equations in the asymptotic regions, but in order for this conservation to be reflected in the Green functions one has to integrate over metrics with a much wider class of asymptotic behaviour.

Despite the fact that they do not have all the required properties, there is reason to believe that the Green functions obtained from these model calculations give the right order of magnitude estimates of the effects of topological fluctuations. The Cauchy data for plane waves $k_{1}$ and $k_{2}$ will be concentrated on null geodesic generators $\lambda_{1}$ and $\lambda_{2}$ of $\mathscr{I}$ with affine parameters $v_{1}$ and $v_{2}$. The integration of this data with the analytic continuation of the free field Green function, $G_{E}\left(x_{1}, x_{2}\right)$ in a given asymptotically Euclidean metric can be regarded as a double contour integral in the complex variables $v_{1}$, and $v_{2}$. The singularities of this integral will occur at the values of $v_{1}$ and $v_{2}$ for which $\lambda_{1}$ and $\lambda_{2}$ can be joined by a null geodesic in the complexified metric. In a general metric there may be many such singularities, but one would expect that the dominant contribution to the contour integral would come from the simplest kind of singularity which are those that occur in the models described above. These give a result of the form

$$
\varrho^{2} J_{2 s}\left(\left[\varrho^{2} k_{1}{ }^{\mu} k_{2}{ }^{\nu} Q_{\mu \nu}\left(\theta_{i}\right)\right]^{1 / 2}\right),
$$




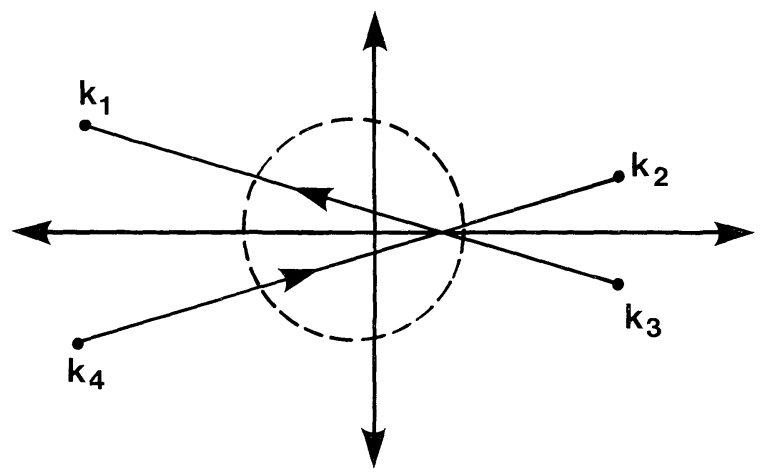

Fig. 5

where $s$ is the spin of the field, $\varrho$ is the typical scale size of the fluctuation and $\theta_{i}$ are dimensionless parameters such as angles which characterize the fluctuation. To obtain a $2 n$-point Green function one has to multiply together $n$ expressions like (5.1), multiply by the gravitational action of the metric (of order $e^{-m_{p}^{2} \varrho^{2}}$ ) and average over $\varrho, \theta_{i}$ and the position of the topological fluctuation. The averaging over the position will give a $\delta$-function in the sum of the momenta. The averaging over $\theta_{i}$ will give a factor of order unity and will conserve angular momentum. The measure for the scale factor, $\varrho$, is not known, but one might expect the dominant contributions come from $\varrho \sim m_{p}^{-1}$, i.e. from fluctuation on the scale of the Planck length. For normal energies $k_{1}$ and $k_{2}$ which are small compared to the Planck energy, $m_{p}$, the argument, $y$, of the Bessel function in (5.1) will be very small compared to 1 . Thus the Bessel function can be approximated by $y^{2 s}$. The integration over the $\theta_{i}$ will then give a Green function of order

$$
\varrho^{2 n(1+s)} K^{1 / 2}\left(k_{1}, \ldots, k_{2 n}\right),
$$

where $K$ is some scalar polynomial constructed out of $2 s$ copies of each momentum, $k_{i}^{\mu}$, the flat space metric, $\eta_{\mu v}$, and the alternating tensor, $\varepsilon_{\mu v \varrho \sigma}$. These Green functions are similar to those that would result from effective interactions of the form

$$
\begin{array}{ll}
m_{p}^{4-2 n}(\phi \bar{\phi})^{n} & \text { for scalar particles } \\
m_{p}^{4-3 n}(\bar{\psi} \psi)^{n} & \text { for spin- } \frac{1}{2} \text { particles } \\
m_{p}^{4-4 n}\left(F_{\mu \nu}\right)^{2 n} & \text { for spin-1 particles }
\end{array}
$$

Thus one can represent them by diagrams with effective vertices of these forms. Unlike ordinary interactions, the positions of the singularities in the complex $\left(v_{1}, v_{2}\right)$ - plane allow them to be non-zero in diagrams for the $\$$ operator which cannot be disconnected into two parts.

The simplest example is shown in Fig. 5, which represents a particle which falls into a quantum gravitational bubble or virtual black hole and comes out as an antiparticle. This diagram will be zero if the particle number is coupled to a long range field like the electromagnetic field because the conservation of the charge for such a field is a consequence of the Maxwell equations in the asymptotic region. 


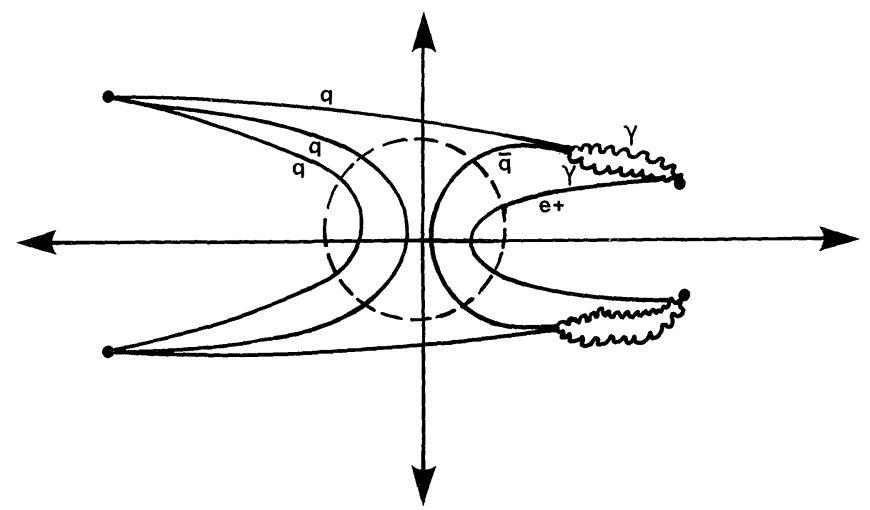

Fig. 6

However the probability of this process can be non-zero if particle number is conserved not because of a local gauge invariance, but only because of some global $U(1)$ symmetry. This will be discussed in Sect. 6 .

In the case of a scalar particle, the probability for this will not be suppressed by any powers of the Planck mass and so will be high. The final state will be mixed with $\operatorname{tr}\left(\varphi^{2}\right)<1$ because there will be non-zero probabilities for finding an antiparticle or for finding the original particle unchanged, but the off-diagonal elements between the particle and antiparticle states will be zero.

In the case of fermions, Fig. 5 would be suppressed by $m_{p}^{-2}$. In fact it would be exactly zero for a free zero rest mass particle because $+k_{1}=+k_{4}=-k_{2}=-k_{3}$ and $k_{1}$ is null. Thus the scalar polynomial, $K$ would be zero. One can get non-zero probabilities for fermions in more complicated diagrams, but these will be suppressed by more powers of $m_{p}$ and so would be very small. An example, the decay of a baryon which would occur by two quarks falling into a bubble and coming out as an antiquark and an electron. The antiquark annihilates with the remaining quark to produce a pair of photons. This is shown in Fig. 6. The lifetime of the baryon to this gravitational decay is of the order

$$
\left(\frac{m_{b}}{m_{p}}\right)^{8} \cdot \frac{1}{m_{b}} \sim 10^{122} \text { years }
$$

This is much longer than the lifetime predicted by grand unified theories which is of the order of $10^{30}$ years if the grand unified mass is about $10^{15} \mathrm{GeV}$.

\section{6. $U(1)$ and CPT Invariance}

Suppose that the Lagrangian of the theory admits a global $U(1)$ invariance

$$
\begin{gathered}
\delta \phi=i \alpha \phi, \\
\delta \phi^{+}=-i \alpha \phi^{+},
\end{gathered}
$$


where $\alpha$ is a constant. Then the current

$$
j^{\mu}=\left(\frac{\delta \mathscr{L}}{\delta \phi_{, \mu}}\right) \frac{\delta \phi}{\delta \alpha}+\left(\frac{\delta \mathscr{L}}{\delta \phi_{, \mu}^{+}}\right) \frac{\delta \phi^{+}}{\delta \alpha}
$$

obeys the conservation law

$$
j_{; \mu}^{\mu}=0 \text {. }
$$

For instance, in the case of complex scalar field, the current is

$$
j_{\mu}=i\left(\phi^{+} \stackrel{\leftrightarrow}{\nabla}_{\mu} \phi\right) \text {. }
$$

In the case of a globally hyperbolic space-time, the conservation law (6.3) implies that the final charge, $Q_{+}$, is equal to the initial charge, $Q_{-}$, where

$$
Q_{ \pm}=\int_{\Sigma_{ \pm}} j_{\mu} d \Sigma^{\mu}
$$

and the integrals are taken over surfaces $\Sigma_{+}$or $\Sigma_{-}$in the asymptotic future or past.

The invariance (6.1) implies that the Green functions will be non-zero only in the case of functions which contain an equal number of $\phi$ operators and of the adjoint operators, $\phi^{+}$. In particular, this implies that the $S$ operator connects states in $\mathscr{H}_{-}$only with states in $\mathscr{H}_{+}$with the same $U(1)$ charge. If asymptotic completeness (Axiom 7) holds, this means that the superscattering operator conserves the $U(1)$ charge, $Q$. However if asymptotic completeness does not hold, there can be non-zero probabilities to go from initial states to final states with a different $U(1)$ charge as in Fig. 5. The reason is that even though the $U(1)$ current is locally conserved by (6.3), the global structure of the space-time may be such that the initial or final surfaces $\Sigma_{-}$and $\Sigma_{+}$are not Cauchy surfaces and hence do not intersect all the current. One can turn the argument the other way round: if a black hole can be formed out of massive particles obeying a global $U(1)$ symmetry and can evaporate and disappear completely with a violation of $U(1)$ charge conservation, then quantum gravity cannot obey asymptotic completeness (Axiom 7).

The decay of pure quantum states into mixed states would seem to imply that quantum gravity has an inbuilt arrow of time. However, this is not the case: one can show that Axiom 1 (Poincaré Invariance), Axiom 2 (Hermiticity), Axiom 4 (Spectral Condition), and Axiom 8 (Euclidean Postulate) imply that the Green functions are invariant under the anti-unitary CPT operator, $\Theta$, i.e.

$$
\left\langle\phi\left(x_{1}\right) \phi^{+}\left(x_{2}\right), \ldots, \phi\left(x_{n}\right)\right\rangle=(-1)^{j}(-i)^{f}\left(\left\langle\phi^{+}\left(-x_{1}\right) \phi\left(-x_{2}\right), \ldots, \phi^{+}\left(-x_{n}\right)\right\rangle\right)^{*},
$$

where $f$ is the number of fermion operators and $j$ is the number of dotted spinor indices. The proof is as follows. The spectral conditions and the Euclidean postulate imply that the Green functions are the boundary values of a holomorphic function $G_{E}\left(x_{1}, \ldots, x_{n}\right)$. Poincaré invariance implies that $G_{E}$ must be invariant under complex Lorentz transformations which are isomorphic to $\operatorname{SL}(2, \mathbb{C}) \times \overline{\mathrm{SL}(2, \mathbb{C})}$. In particular, they must be invariant under the transformation $(1,-1)$ which will send a point $x_{i} \rightarrow-x_{i}$ and will change the sign of quantities with dotted spinor indices. Thus

$$
\left\langle\phi\left(x_{1}\right) \phi^{+}\left(x_{2}\right), \ldots, \phi\left(x_{n}\right)\right\rangle=(-1)^{j}(-i)^{f}\left\langle\phi\left(-x_{n}\right), \ldots, \phi^{+}\left(-x_{2}\right) \phi\left(-x_{1}\right)\right\rangle .
$$


The reason that the operator ordering is reversed in (6.7) is that the operator ordering is obtained by analytically continuing $G_{E}\left(x_{1}, \ldots, x_{n}\right)$ with a definite ordering of the imaginary time coordinate of the points $x_{1}, \ldots, x_{n}$. The transformation $x_{i} \rightarrow-x_{i}$ reverses this ordering and hence reverses the operator ordering. The Hermiticity condition implies

$$
\begin{aligned}
& \left\langle\phi\left(x_{1}\right) \phi^{+}\left(x_{2}\right), \ldots, \phi\left(x_{n}\right)\right\rangle=(-1)^{j}(-i)^{f}\left\langle\phi\left(-x_{n}\right), \ldots, \phi^{+}\left(-x_{2}\right) \phi\left(-x_{1}\right)\right\rangle \\
& =(-1)^{j}(-i)^{f}\left(\left\langle\phi^{+}\left(-x_{1}\right) \phi\left(-x_{1}\right), \ldots, \phi\left(-x_{n}\right)\right\rangle\right)^{*} .
\end{aligned}
$$

The arrow of time in the decay of pure quantum states comes, not from the Green functions themselves, but from the way that they are used to calculate the $\$$ operator element which gives the probability of going from the initial state $|A\rangle\langle B|$ to the final state $|C\rangle\langle D|$

$$
\begin{gathered}
\$_{C A}^{D} B=\underbrace{\left\langle a_{-}\left(k_{1}\right), \ldots, a_{-}\left(k_{m}\right)\right.}_{B} \underbrace{a_{+}^{+}\left(k_{1}^{\prime}\right), \ldots, a_{+}^{+}\left(k_{m^{\prime}}^{\prime}\right.}_{C}) \\
\underbrace{a_{+}\left(l_{1}^{\prime}\right), \ldots, a_{+}\left(l_{n^{\prime}}^{\prime}\right)}_{D} \underbrace{\left.a_{-}^{+}\left(l_{1}\right), \ldots, a_{-}^{+}\left(l_{n}\right)\right\rangle}_{A}
\end{gathered}
$$

By CPT invariance, (3.8) is equal to

$$
\begin{gathered}
(-1)^{j}(-i)^{f}\left(\left\langleb_{+}\left(-k_{1}\right), \ldots, b_{+}\left(-k_{m}\right) b_{-}^{+}\left(-k_{1}^{\prime}\right), \ldots, b_{-}^{+}\left(-k_{m^{\prime}}^{\prime}\right)\right.\right. \\
\left.\left.b_{-}\left(-l_{1}^{\prime}\right), \ldots, b_{-}\left(-l_{n^{\prime}}^{\prime}\right) b_{+}^{+}\left(-l_{1}\right), \ldots, b_{+}^{+}\left(-l_{n}\right)\right\rangle\right)^{*} .
\end{gathered}
$$

In other words, all annihilation and creation operators are replaced by the corresponding antiparticle operators, all spatial momenta are reversed and the future and the past are interchanged. However, (6.9) is not related to the $\$$ operator element expressing the probability of going from the CPT inverse of the state $|C\rangle\langle D|$ to the CPT inverse of the state $|A\rangle\langle B|$. This latter probability is

$$
\begin{gathered}
\left\langle b_{-}\left(-l_{1}^{\prime}\right), \ldots, b_{-}\left(-l_{n^{\prime}}^{\prime}\right) b_{+}^{+}\left(-l_{1}\right), \ldots, b_{+}^{+}\left(-l_{n}\right)\right. \\
\left.b_{+}\left(-k_{1}\right), \ldots, b_{+}\left(-k_{m}\right) b_{-}^{+}\left(-k_{1}^{\prime}\right), \ldots, b_{-}^{+}\left(-k_{m^{\prime}}^{\prime}\right)\right\rangle .
\end{gathered}
$$

The operator ordering in (6.10) is different from that in (6.9): the annihilation and creation operators in the past appear on the right and the left sides of (6.10), but they are in the centre of (6.9).

It may well be true that (6.10) equals (6.9) modulo possible sign and complex conjugation. In this case one can say the superscattering operator obeys weak CPT invariance or detailed balance [3-5]. This means that the probability of going from the state $|A\rangle\langle B|$ to the state $|C\rangle\langle D|$ is the complex conjugate of the probability of going from the CPT inverse of $|C\rangle\langle D|$ to the CPT inverse of $|A\rangle\langle B|$. One can also define a strong sense of CPT invariance for the $\$$ operator which requires that if an initial density matrix, $\varrho_{-}$, gives rise to a final density matrix, $\varrho_{+}$, then the initial density matrix, $\Theta \varrho_{+} \Theta$ gives rise to $\Theta \varrho_{-} \Theta$. It can be shown that strong CPT invariance implies that the $\$$ operator can be factorized into an $S$ operator, and its adjoint, and hence asymptotic completeness holds $[4,5]$. 
An example of how the $\$$ operator can fail to exhibit the strong CPT invariance even though the Green functions are CPT invariant has been discussed by Page [13]. Suppose, as in the case of black hole evaporation, that one can find a total Hilbert space $\mathscr{H}$ which is a tensor product of the future Hilbert space $\mathscr{H}_{+}$with another unobserved Hilbert space $\mathscr{H}_{+}^{\prime}$. In order to be time symmetric one should also assume that $\mathscr{H}$ is the tensor product of the past Hilbert space $\mathscr{H}_{-}$with another unobserved Hilbert space $\mathscr{H}_{-}^{\prime}$.

A state $|\lambda\rangle$ in $\mathscr{H}$ may be represented in terms of its components in $\mathscr{H}_{-} \otimes \mathscr{H}_{-}^{\prime}$ :

$$
|\lambda\rangle=\lambda^{A B}\left|A_{-}\right\rangle\left|B_{-}^{\prime}\right\rangle .
$$

Similarly,

$$
|\lambda\rangle=\lambda_{+}{ }^{C D}\left|C_{+}\right\rangle\left|D_{+}^{\prime}\right\rangle \text {. }
$$

These components will be related by a unitary operator $U$,

$$
\lambda_{+}{ }^{C D}=U_{A B}^{C D} \lambda_{-}{ }^{A B} .
$$

The vacuum state $|0\rangle$ can be represented by $\left|0_{-}\right\rangle\left|0_{-}^{\prime}\right\rangle$ or by $\left|0_{+}\right\rangle\left|0_{+}^{\prime}\right\rangle$, where $\left|0_{-}^{\prime}\right\rangle$ and $\left|0_{+}^{\prime}\right\rangle$ are vacuum states for $\mathscr{H}_{-}^{\prime}$ and $\mathscr{H}_{+}^{\prime}$, respectively.

In the definition of the superscattering operator $\$$, one acts with creation and annihilation operators in the past and to form the state $\left|A_{-}\right\rangle\left|0_{-}^{\prime}\right\rangle\left\langle B_{-}\right|\left\langle 0_{-}^{\prime}\right|$. In terms of $\mathscr{H}_{+} \otimes \mathscr{H}_{+}^{\prime}$ this will be

$$
U_{A 0}^{C D}\left|C_{+}\right\rangle\left|D_{+}^{\prime}\right\rangle\left\langle E_{+}\right|\left\langle F_{+}^{\prime}\right|\left(U_{E F}^{B 0}\right)^{*} .
$$

When one sums over the unobserved states on $\mathscr{H}_{+}^{\prime}$, one obtains

$$
\$_{E A}^{C}{ }^{B}=U_{A 0}^{C D}\left(U_{E D}^{B 0}\right)^{*} .
$$

The time asymmetry in the decay of a pure state arises because we have prepared the system in a particular state $\left|A_{-}\right\rangle\left|0_{-}^{\prime}\right\rangle\left\langle B_{-}\right|\left\langle 0_{-}^{\prime}\right|$ by acting with creation and annihilation operators in the past, but we have observed it in the future by a measurement only on $\mathscr{H}_{+}$. In general, the state in the future will not be of the form

$$
\varrho_{+}{ }_{D}{ }_{D}\left|C_{+}\right\rangle\left|0_{+}^{\prime}\right\rangle\left\langle D_{+}\right|\left\langle 0_{+}^{\prime}\right| \text {. }
$$

However the state (6.16) is what one would produce if one was to try to prepare the state $\varrho_{+}{ }^{C}$ in the future by acting with creation and annihilation operators in the future. Thus the time asymmetry in the decay of pure states into mixed states is a reflection of the way we interpret the theory rather than a property of the theory itself. Nevertheless, the unpredictability which it introduces seems to be a necessary feature of any theory of quantum gravity which allows the metric to fluctuate from being globally hyperbolic. 


\section{References}

1. Hawking, S.W.: Particle creation by black holes. Commun. Math. Phys. 43, 199-220 (1975)

2. Gross, D.J., Perry, M.J., Jaffe, L.G. Instability of flat space at finite temperature. Phys. Rev. D25, 330-355 (1982)

3. Hawking, S.W.: Breakdown of predictability in gravitational collapse. Phys. Rev. D14, 2460 (1976)

4. Page, D.N.: Is black-hole evaporation predictable. Phys. Rev. Lett. 44, 301 (1980)

5. Wald, R.M.: Black holes, thermodynamics, and time reversibility. In: Quantum gravity. 2. Isham, C.J., Penrose, R., Sciama, D.W., Oxford, Clarendon Press 1981

6. Penrose, R.: Zero rest-mass fields including gravitation, asymptotic behaviour. Proc. R. Soc. Lond. A 284, 159-203 (1965)

7. Sachs, R.K.: Asymptotic symmetries in gravitational theory. Phys. Rev. 128, 2851 (1962)

8. Penrose, R.: Asymptotic properties of fields and space-times. Phys. Rev. Lett. 10, 66-68 (1963)

9. Hawking, S.W., Ellis, G.F.R.: The large scale structure of space-time. Cambridge: Cambridge University Press 1973

10. Streater, R.F., Wightman, A.S. : PCT, spin and statistics, and all that, New York: Benjamin 1964

11. Hawking, S.W., Page, D.N., Pope, C.N.: Quantum gravitational bubbles. Nucl. Phys. B. 170, 283 (1980)

12. Warner, N.P.: The scattering of spin-1 particles by quantum gravitational bubbles. Commun. Math. Phys. 86, 419-436 (1982)

13. Page, D.N. : Is quantum gravity time symmetric and/or deterministic? GRG 14, 299-302 (1982)

Communicated by A. Jaffe

Received July 21, 1982 
